\title{
Integrated genomics approach to identify biologically relevant alterations in fewer samples
}

\author{
Pratik Chandrani', Pawan Upadhyay ${ }^{1}$ Prajish lyer ${ }^{1}$, Mayur Tanna', Madhur Shetty ${ }^{1}$, Gorantala Venkata Raghuram , \\ Ninad Oak', Ankita Singh', Rohan Chaubal', Manoj Ramteke', Sudeep Gupta² and Amit Dutt ${ }^{\text {** }}$ (D)
}

\begin{abstract}
Background: Several statistical tools have been developed to identify genes mutated at rates significantly higher than background, indicative of positive selection, involving large sample cohort studies. However, studies involving smaller sample sizes are inherently restrictive due to their limited statistical power to identify low frequency genetic variations.

Results: We performed an integrated characterization of copy number, mutation and expression analyses of four head and neck cancer cell lines - NT8e, OT9, AW13516 and AW8507- by applying a filtering strategy to prioritize for genes affected by two or more alterations within or across the cell lines. Besides identifying TP53, PTEN, HRAS and MET as major altered HNSCC hallmark genes, this analysis uncovered 34 novel candidate genes altered. Of these, we find a heterozygous truncating mutation in Nuclear receptor binding protein, NRBP1 pseudokinase gene, identical to as reported in other cancers, is oncogenic when ectopically expressed in $\mathrm{NIH}-3 \mathrm{~T} 3$ cells. Knockdown of NRBP1 in an oral carcinoma cell line bearing NRBP1 mutation inhibit transformation and survival of the cells.

Conclusions: In overall, we present the first comprehensive genomic characterization of four head and neck cancer cell lines established from Indian patients. We also demonstrate the ability of integrated analysis to uncover biologically important genetic variation in studies involving fewer or rare clinical specimens.
\end{abstract}

\section{Background}

Head and neck squamous cell carcinoma (HNSCC) is the sixth-most-common cancer worldwide, with about 600,000 new cases every year, and includes cancer of the nose cavity, sinuses, lips, tongue, mouth, salivary glands, upper aerodigestive tract and voice box [1]. Recent large scale cancer genome sequencing projects have identified spectrum of driver genomic alterations in HNSCC including CDKN2A, TP53, PIK3CA, NOTCH1, HRAS, FBXW7, PTEN, NFE2L2, FAT1, and CASP8 [2-4]. These landmark studies apply elegant statistical methodologies like MutSig [5], Genome MuSiC [6], Intogen [7], InVEx [8], ActiveDrive [9] and GISTIC [10] in identifying significantly altered genes across large sample cohorts by comparing rate of mutations of each gene with

\footnotetext{
*Correspondence: adutt@actrec.gov.in

${ }^{1}$ Advanced Centre for Treatment, Research and Education in Cancer, Tata Memorial Center, Navi Mumbai, Maharashtra 410210, India

Full list of author information is available at the end of the article
}

background mutation rate to determine an unbiased enrichment- a minimum 150 patients or higher is required for identification of somatic mutations of $10 \%$ population frequency in HNSCC [11]. These genomewide analysis may not be directly applicable for studies involving fewer or rare clinical specimen that are inherently restrictive due to the limited statistical power to detect alterations existing at lower frequency.

On the other hand, given that a cancer gene could be selectively inactivated or activated by multiple alterations, an integrative study design performed by combining multiple data types can potentially be helpful to achieve the threshold for statistical significance for studies involving fewer or rare clinical specimen. For example, a tumor suppressor gene- deleted in $1 \%$ of patients, mutated in another $3 \%$, promoterhypermethylated in another $2 \%$ and out of frame fused with some other chromosomal region in $2 \%$ - may be considered to be altered with a cumulative effect of $8 \%$ 
based on integrative analysis [12, 13]. Combinatorial sources of genetic evidence converging at same gene or signalling pathway can also limit false positives by filtering strategy and potentially reducing the multiple hypothesis testing burden for identification of causal genotype-phenotype associations [14]. Using similar approaches for posterior refinement to indicate positive selection, Pickering et al. identified four key pathways in oral cancer by integrating methylation to copy number variation and expression [15]; and, more recently, Wilkerson et al. proposed superior prioritisation of mutations based on integrated analysis of the genome and transcriptome sequencing than filtering based on conventional quality filters [16]. These and several other reports all together emphasize integration of multiplatform genomic data for identification of cancer related genes [17].

Here, we perform characterization of four head and neck cancer cell lines, established from Indian head and neck cancer patients, using classical cytogenetic approach, SNP arrays, whole exome and whole transcriptome sequencing. Next, we apply the widely used posterior filtering strategy of results obtained from genome wide studies to effectively reduce the amount of data obtained from individual platforms. Adopting such an integrative approach allow us to identify biological relevant alterations affected by two or more events even from fewer samples.

\section{Methods}

\section{Cell culturing and single cell dilution for establishing clonal cells}

Four HNSCC tumor cell lines established within Tata Memorial Center from Indian patients and described before were acquired: NT8e, OT9, AW13516, AW8507 $[18,19]$. All the cell lines were maintained in DMEM media (Gibco, USA). For clonal selection, growing culture was trypsinized and diluted as 1 cell per $100 \mathrm{ml}$ of media and dispensed in a 96 well plate with follow up subculture of clones that survived.

\section{SNP array analysis}

Genomic DNA was extracted from pre-clonal and clonal cell lines using PAXgene Tissue DNA Kit (Qiagen, USA). $200 \mathrm{ng}$ of good quality DNA from each sample was submitted to Sandor Proteomics (Hyderabad, India) for sample preparation and genome wide SNP array using Illumina Infinium assay (Human660W-quad BeadArray chip) following manufacturer's standard protocol. Array data was pre-processed using GenomeStudio (Illumina Inc., USA) for quality control check. To retain only good quality genotyping calls, a threshold GenCall score of 0.25 was used across all samples. A total of 396, 266 SNPs were retained after this filtering. These SNPs were then used for copy number analysis using Genome Studio plugin cnvPartition 3.2 and an $\mathrm{R}$ package Genome Alteration Print (GAP) [20]. Inferred copy numbers were then annotated with genomic features using BedTools (v. 2.17.0) [21]. Copy number segments of more than $10 \mathrm{Mb}$ in size were classified as arm-level amplifications and were identified as non-significant alterations. Focal amplifications (less than $10 \mathrm{Mb}$ ) were used for further analysis.

\section{Cytogenetic karyotyping}

Cells grown in complete media (60-70\% confluent) were treated with colcemid ( $0.1 \mathrm{ng} / \mathrm{ul}$, Sigma, USA) to arrest them in metaphase. After incubation of $6 \mathrm{~h}$ at $37 \mathrm{oC}$ and trypsinisation, cells were washed with prewarmed $\mathrm{KCl}(0.075 \mathrm{M})$ (Sigma, USA) and incubated with $\mathrm{KCl}$ at $37^{\circ} \mathrm{C}$ in water bath for $60 \mathrm{~min}$. After the incubation is over, cells were fixed with Carnoy's fixative solution on pre chilled microscopic glass slides (chilled in alcohol) by pipette around $70 \mu \mathrm{l}$ of cell suspension, drop by drop from height $(50 \mathrm{~cm})$. Slides were kept on the water bath at $70{ }^{\circ} \mathrm{C}$ for few seconds followed by drying on heating block (set at $80{ }^{\circ} \mathrm{C}$ ). Metaphase of cells was confirmed by observing chromosomes using a phase contrast inverted microscope (Zeiss, USA). Confirmed metaphase captured cells were aged by keeping the slides at $60{ }^{\circ} \mathrm{C}$ for $3 \mathrm{~h}$ followed by trypsin digest (Trypsin/EDTA - concentration of $0.025 \%$, Sigma, USA). Giemsa stain (Sigma, USA) (3\%) was applied using coplin Jar for 15 min on slides followed by washing with distilled water.

\section{Exome sequencing}

Exome enrichment was performed using manufacturer's protocol for Illumina TruSeq exome enrichment kit in which $500 \mathrm{ng}$ of DNA libraries from six samples were pooled to make total $3 \mu \mathrm{g}$ DNA mass from which $62 \mathrm{MB}$ of targeted exonic region covering 20,976 genes was captured. Exome enriched library was quantified and validated by real-time PCR using Kappa quantification kit at the Next-Generation Genomics Facility (NGGF) at Center for Cellular and Molecul ar Platforms (CCAMP, India). Whole exome libraries of AW13516, AW8507 and OT9 were loaded onto Illumina HiSeq 1000 for $2 \times 100$ bp paired-end sequencing with expected coverage of $\sim 100 \mathrm{X}$. NT8e cell line was sequenced with $2 \times 54$ bp paired-end and $2 \times 100$ bp paired-end sequencing. Raw sequence reads generated were mapped to NCBI human reference genome (build GRCh37) using BWA v. 0.6.2 [22]. Mapped reads were then used to identify and remove PCR duplicates using Picard tools v. 1.74 (http:broadinstitute.github.io/picard/). Base quality score recalibration and indel re-alignment were performed and variants were called from each cell line separately using GATK v. 1.6-9 [23, 24] and MuTect 
v. 1.0.27783 [25]. All the variants were merged and dumped into local MySQL database for advanced analysis and filtering. We used hard filter for removing variants having below $5 \mathrm{X}$ coverage to reduce false positives. For cell lines we use dbSNP (v. 134) [26] as standard known germline variants database and COSMIC (v. 62) [27] as standard known somatic variants database. Variants identified in cell lines, which are also there in dbSNP but not in COSMIC were subtracted from the database. Remaining variants were annotated using Oncotator (v. 1.0.0.0rc7) [28], and three functional prediction tools PolyPhen2 (build r394) [29], Provean (v. 1.1) [30] and MutationAccessor (release 2) [31]. Variants found deleterious by any two out of three tools were prioritized. Variants having recurrent prediction of deleterious function were prioritized. Variants from exome sequencing were compared to variants identified from transcriptome sequencing for cross-validation using in-house computer program.

\section{Transcriptome sequencing}

Transcriptome libraries for sequencing were constructed according to the manufacturer's protocol. Briefly, mRNA was purified from $4 \mu \mathrm{g}$ of intact total RNA using oligodT beads (TruSeq RNA Sample Preparation Kit, Illumina). 7 pmol of each library was loaded on Illumina flow cell (version 3) for cluster generation on cBot cluster generation system (Illumina) and clustered flow cell was transferred to Illumina HiSeq1500 for paired end sequencing using Illumina paired end reagents TruSeq SBS Kit v3 (Illumina) for 200 cycle. De-multiplexing was done using CASAVA (version 1.8.4, Illumina). Actively expressed transcripts were identified from sequencing data by aligning them to the reference genome hg19 using Tophat (v. 2.0.8b) [32] and quantifying number of reads per known gene using cufflinks (v.2.1.1) pipeline [33]. All the transcripts were then binned by $\log _{10}(\mathrm{FPKM}+1)$ to differentiate the significantly expressed transcripts from the background noise. Since paired normal of these cell lines cannot be obtained, we defined significant change in expression for those genes whose expression is higher $(>60 \%)$ or lower $(<40 \%)$ than the median expression as suggested in [34]. Gene set enrichment was performed by submitting actively expressed transcripts lists to MSigDB V4 [35] and filtering resulting gene lists by $p$-value of enrichment. Variants were identified from transcriptome sequencing using GATK $[23,24]$. Only variants having overlap with exome sequencing were considered as true genomic variants. Fusion transcripts were identified using ChimeraScan (v.0.4.5) [36]. Candidate fusion events supported by minimum 10 read pairs were used for integration and visualization in Circos plot.

\section{Integrated analysis}

Genes identified to be altered by SNP array, transcriptome sequencing and exome sequencing were then used for integrative analysis to prioritize the genes which are harbouring multiple types of alteration in same or different cell line. Gene level converging of genomic data were emphasized in identification of biologically relevant alterations across platform and samples. Taking this into consideration, we designed gene prioritization based on three steps: 1) selection of genes harbouring positive correlation of focal copy number and gene expression; 2) selection of genes harbouring point mutations with detectable transcript and or altered copy number, and 3) selection of genes harbouring multiple type of alterations identified from above two gene lists (Additional file 1: Figure S7). Circos plot representation of integrated genomics data was generated using Circos tool (v. 0.66) [37].

\section{Sanger sequencing validation}

PCR products were purified using NucleoSpin Gel and PCR Clean-up kit (MACHEREY-NAGEL) as per manufacture's protocol and quantified using Nano-Drop 2000c Spectrophotometer (Thermo Fisher Scientific Inc.) and submitted for sequencing in capillary electrophoresis 3500 Genetic Analyzer (Life Technologies). Sanger sequencing traces were analysed for mutation using Mutation Surveyor [38]. The details of all the primers used for mutation analysis have been provided in Additional file 2: Table S7.

\section{DNA copy number validation}

Quantitative-real time PCR and data analysis was performed using Type-it ${ }^{\circ}$ CNV SYBR ${ }^{\circ}$ Green PCR (cat. No. 206674) as per manufacturer's instructions on $7900 \mathrm{HT}$ Fast Real-Time PCR System. The details of all the primers used for DNA copy number analysis have been provided in Additional file 2: Table S8.

\section{RNA extraction, CDNA synthesis, quantitative real time PCR}

Total RNA was extracted from cell lines using RNeasy RNA isolation kit (Qiagen) and Trizol reagent (Invitrogen) based methods and later resolved on $1.2 \%$ Agarose gel to confirm the RNA integrity. RNA samples were DNase treated followed (Ambion) by first strand cDNA synthesis using Superscript III kit (Invitrogen) and semi-quantitative evaluative PCR for GAPDH was performed to check the cDNA integrity. cDNA was diluted 1:10 and reaction was performed in $10 \mu \mathrm{l}$ volume in triplicate. The melt curve analysis was performed to check the primer dimer or non-specific amplifications. Real-time PCR was carried out using KAPA master mix (KAPA SYBR ${ }^{\circ}$ FAST Universal q PCR kit) as per manufacturer's instructions in triplicate on 
7900HT Fast Real-Time PCR System. All the experiments were repeated at least twice independently. The data was normalized with internal reference GAPDH, and analysed by using delta-delta $\mathrm{Ct}$ method described previously [39]. The details of all the primers used for expression analysis have been provided in Additional file 2: Table S8.

\section{Generation of $\mathrm{p}$ BABE-NRBP1-PURO constructs}

The cDNA of Human NRBP1 was amplified from AW13516 cell line using Superscript III (Invitrogen, cat no 18080-093) in a TA cloning vector pTZ57R/ $\mathrm{T}$ (InsTAclone PCR cloning kit, K1214, ThermoScientific), later site-directed mutagenesis was done using QuikChange II Site-Directed Mutagenesis Kit (cat.no. 200523) as per manufacturer's instructions. Later both wild type and mutant NRBP1 cDNA sequenced confirmed using Sanger sequencing and were sub-cloned in to retroviral vector $p$ BABE-puro using restriction digestion based cloning (SalI and BamHI).

\section{Generation of stable clone of $\mathrm{NIH}-3 \mathrm{~T} 3$ overexpressing NRBP1}

Two hundred ninety-three T cells were seeded in 6 well plates one day before transfection and each constructs (pBABE-puro) along with pCL-ECO helper vector were transfected using Lipofectamine LTX reagent (Invitrogen) as per manufacturer's protocol. Viral soup was collected 48 and $72 \mathrm{~h}$ post transfection, passed through $0.45 \mu \mathrm{M}$ filter and stored at $4{ }^{\circ} \mathrm{C}$. Respective cells for transduction were seeded one day before infection in six well plate and allowed to grow to reach 50-60\% confluency. One $\mathrm{ml}$ of the virus soup (1:5 dilution) and $8 \mathrm{ug} / \mathrm{ml}$ of polybrene (Sigma) was added to cells and incubated for six hours. Cells were maintained under puromycin (Sigma) selection.

\section{shRNA mediated knockdown of NRBP1 in HNSCC cells}

We retrieved shRNA sequences targeting human NRBP1 from TRC (The RNAi Consortium) library database located in sh1 (3' UTR) and sh2 (CDS). Target sequences of NRBP1 shRNA constructs: sh1 (TRCN0000001437), 5' -CCCTCTGCACTTTGTTTACTTCT-3'; sh2 (TRCNO 000001439), 5' -TGTCGAGAAGAGCAGAAGAATCT-3'. shGFP target sequences is $5^{\prime}$-GCAAGCTGACCCT GAAGTTCAT-3'. p-LKO.1 GFP shRNA was a gift from David Sabatini (Addgene plasmid \# 30323) [40]. Cloning of shRNA oligos were done using AgeI and EcoRI restriction site in p-LKO.1 puro constructs. Bacterial colonies obtained screened using PCR and positive clone were sequence verified using Sanger sequencing. Lentiviral production and stable cell line generation performed as described earlier [41]. In brief, Lentivirus were produced by transfection of shRNA constructs and two helper vector in $293 \mathrm{~T}$ cells as described [42]. Transduction was performed in HNSCC cells by incubating for $6 \mathrm{~h}$ in presence of $10 \mu \mathrm{g} / \mathrm{ml}$ polybrene and post infection media was replaced with fresh media. Puromycin selection was performed two days post infection in presence of $1 \mu \mathrm{g} / \mathrm{ml}$. Puromycin selected cells were harvested and total cell lysate prepared and expression of NRBP1 was analysed using anti-NRBP1 antibody (Santa Cruz Biotechnology; sc-390087) and GAPDH (Santa Cruz Biotechnology; sc-32233).

\section{Soft Agar colony formation assay}

The cells were harvested $48 \mathrm{~h}$ after transfection, and an equal number of viable cells were plated onto soft agar after respective treatments for determination of anchorage-independent growth. For analysis of growth in soft agar, $5 \times 10^{3}$ cells were seeded in triplicate onto a six well dish (Falcon) in $3 \mathrm{ml}$ of complete medium containing $0.33 \%$ agar solution at $37{ }^{\circ} \mathrm{C}$. Cells were fed with $500 \mu \mathrm{l}$ of medium every 2 days. From each well randomly 10 field images were taken using Phase contrast Inverted microscope (Zeiss axiovert $200 \mathrm{~m}$ ) and colonies were counted manually.

Growth curve analysis - 25,000 cells/well were seeded in 24 well plates and growth was assessed post day 2, 4 and 6 by counting the cells using a haemocytometer. Percent survival were plotted at day 4 relative to day 2 and later normalized against scrambled or empty vector control.

\section{Western blot analysis}

Cells were lysed in RIPA buffer and protein concentration was estimated using BCA method [43]. 50 and $100 \mu \mathrm{g}$ protein was used for NIH-3 T3 and HNSCC cell lines western analysis. The protein was separated on $10 \%$ SDS-PAGE gel, transfer was verified using Ponceau $S$ (Sigma), transferred on nitrocellulose membrane and blocked in Tris-buffered saline containing $5 \%$ BSA (Sigma) and $0.05 \%$ Tween-20(Sigma). Later, blots were probed with anti-NRBP1 (Santa Cruz Biotechnology; sc-390087), anti-total ERK1/2 (Cell signaling; 4372S), anti-Phospho ERK1/2 (Cell signaling; 4370S) and anti- GAPDH antibody (Santa Cruz Biotechnology; SC-32233). The membranes were then incubated with corresponding secondary HRP-conjugated antibodies (Santa Cruz Biotechnology, USA) and the immune complexes were visualized by Pierce ECL (Thermo Scientific, USA) according to manufacturer's protocol. Western blot experiments were performed as independent replicates.

\section{Statistical analysis}

Chi-square and $\mathrm{t}$-test were performed using $\mathrm{R}$ programming language and GraphPad Prism. A $p$-value cut-off of 0.05 was used for gene expression, copy number and variant analysis. 


\section{Availability of supporting data}

All genomics data have been deposited at the ArrayExpress (http://www.ebi.ac.uk/arrayexpress/), hosted by the European Bioinformatics Institute (EBI), under following accession numbers: E-MTAB-3958 : Whole transcriptome data; E-MTAB-3961 : Whole exome data; and, E-MTAB3960 : SNP array data

\section{Results}

We characterized genetic alterations underlying four head and neck cancer cell lines followed by TCGA dataset to identify cumulative significance of biologically relevant alterations by integrating copy number, expression and point mutation data.

\section{Characterization of four HNSCC cell lines established from Indian patients}

Given that higher accumulative effect of individual genes can be reckoned by integrative analysis, we argue that these alteration can possibly be determined even with fewer samples. As a proof of principle, we performed an integrated characterization of karyotype analysis, copy number analysis, whole transcriptome and exome sequencing of 4 HNSCC cell lines established from Indian patients. In brief, significantly altered chromosomal segments based on copy number analysis were filtered based on nucleotide variant information and aberrant expression of transcripts to allow prioritization of regions harboring either deleterious mutation or expressing the transcript at significantly high levels, in addition to the stringent intrinsic statistical mining performed for each sample.

\section{Karyotype analysis}

The hyperploidy status of AW13516, AW8507, NT8E and OT9 cell lines were inferred by classical karyotyping with an average ploidy of 62, 62, 66 and 64, respectively that were largely consistent with ploidy as inferred form SNP array analysis (Fig. 1a; Additional file 1: Figure S1) and as reported for tumor cells lines [18, 19]. We specifically observed dicentric and ring chromosomes at elevated frequency indicating higher chromosomal instability (CIN) [44]. Overall distribution of chromosomal aberrations in each HNSCC cell lines showed similar pattern, representing an overall similar genomic structure of all HNSCC cell lines.

\section{Copy number analysis}

We performed genotyping microarray using Illumina $660 \mathrm{~W}$ quad SNP array chips of all the cell lines (Additional file 1: Figure S2). After stringent filtering of initial genotyping calls, on an average, 253 genomic segments of copy number changes were obtained per cell line. By limiting segment size at $10 \mathrm{Mb}$ an average 166 focal segments were identified, including loss of copy number and LOH at 3p which is known to have correlation to advanced stage of tumor progression and poor clinical outcome [45, 46]; copy number gain on 11q known to be associated with advanced stage, recurrence and poor clinical outcome [47]; LOH at $8 \mathrm{p}$ and $9 \mathrm{p}$ which are known to be associated with advanced stage and survival [48] (Additional file 2: Table S1); and amplification of known oncogenes EGFR in AW13516, OT9; MYC in AW13516 and AW8507 cells; JAK1 in NT8E, AW8507; NSD1 in AW8507; and MET in AW13516 and OT9 (Additional file 2: Table S2). Several hallmark genes were found to be amplified in cell lines such as CCND1, NOTCH1, and HES1 in all four cells; PIK3CA in AW13516, AW8507; deletion of CDKN2A in AW13516; FBXW7 in NT8E, AW13516 and OT9 cells were detected and validated by real time PCR (Fig. 1b, Additional file 2: Table S2) in each cell line.

\section{Whole transcriptome analysis}

Whole transcriptome sequencing revealed 17,067, 19,374, 16,866 and 17,022 genes expressed in AW13516, AW8507, NT8e and OT9 respectively. Total $~ 5000$ transcripts having less than $0.1 \log _{10}(\mathrm{FPKM}+1)$ were filtered out because of biologically non-significant expression level (Additional file 1: Figure S3). The upper quartile (>60\%) was considered as highly expressed genes and lower quartile $(<40 \%)$ was considered as lowly expressed genes. Gene set enrichment analysis of upper quartile showed enrichment of genes (data not shown) known to be up regulated in nasopharyngeal carcinoma [49]. All the transcripts showed $75 \%$ overlap of expression profile with each other (Additional file 1: Figure S4) indicating overall similar nature of cell lines. Over expression of hallmark of HNSCC such as CCND1, MYC, MET, CTNNB1, JAK1, HRAS, JAG1, and HES1 and down regulation of $F B X W 7, S M A D 4$ in at least 3 cell line were observed and validated by quantitative real time PCR (Additional file 2: Table S3). A positive correlation was observed between transcriptome FPKM and qPCR $\mathrm{Ct}$ values (Fig. 1c).

\section{Analysis of mutational landscape}

All the cell lines were sequenced for whole exome at about $80 \mathrm{X}$ coverage using Illumina HiSeq. The relative coverage of each coding region was comparable across all four cell lines (Additional file 2: Table S4; Additional file 1: Figure S5). The coding part of the four cell line genome consist 28813, 47892, 20864 and 25029 variants in AW13516, AW8507, NT8e and OT9 cell line, respectively. Filtering of known germline variants (SNPs) and low quality variants left 5623, 4498, 2775, 5139 nonsynonymous variants in AW13516, AW8507, NT8e and OT9 cell line, respectively (Additional file 2: Table S4). Of 20 HNSCC hallmark variants predicted as deleterious 

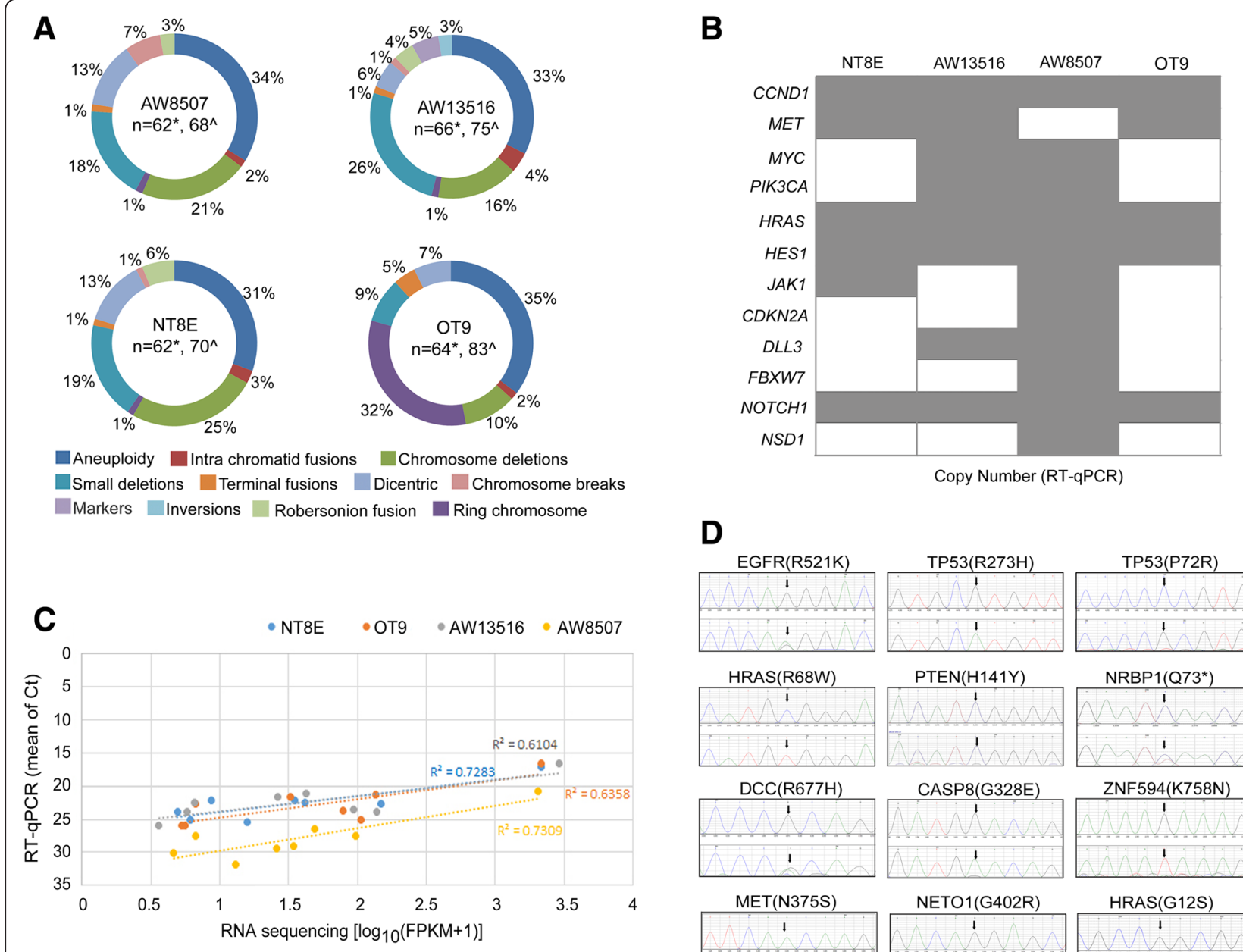

D

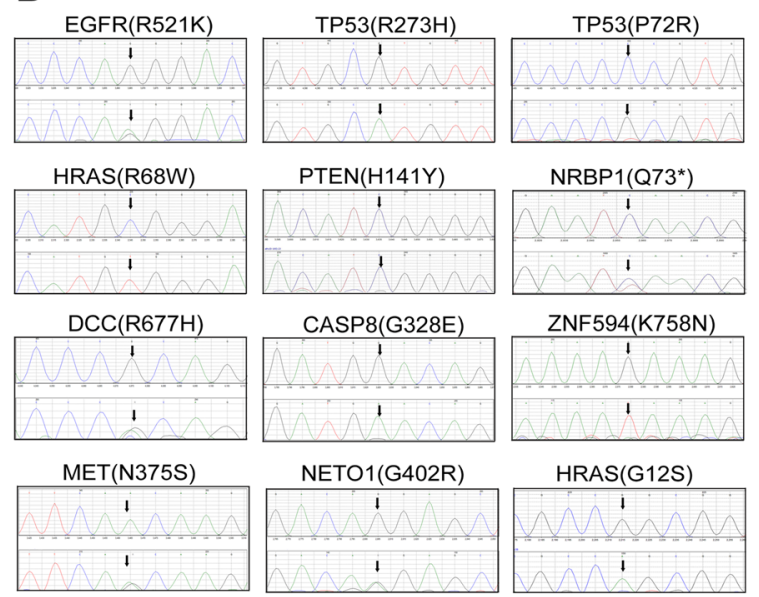

Fig. 1 Genomic alterations identified in HNSCC cell lines. a Chromosomal aberrations identified by 25 independent karyotype of each cell line is represented in circular form. Chromosome numbers in each cell line are indicated by $n$, as observed from karyotype $\left.*^{*}\right)$ and predicted by SNP array $(\wedge)$. $\mathbf{b}$ Heatmap representation of copy number changes in key hallmark genes identified by SNP array and validated by qPCR. Grey box indicates validated and white box indicates invalidated copy number change in respective cell line. c Scatter plot representation of hallmark gene's expression. X-axis shows gene expression quantified by RNA sequencing and Y-axis shows gene expression quantified by RT-qPCR. Genes showed positive correlation between RNA sequencing and RT-qPCR. $\mathbf{d}$ Several high-quality point mutations identified by exome sequencing were validated by Sanger sequencing. Sanger sequencing trace were visualized using Mutation Surveyor software showing reference sequence trace in upper panel and mutant sequencing trace in below panel. Arrows represent mutation position with reference and mutated base indicated above the arrow

by two of three algorithms used for functional prediction [29-31], 17 variants could be validated by Sanger sequencing (Fig. 1d; Additional file 2: Table S5) including: TP53 (R273H), TP53 (P72R), PTEN (H141Y), EGFR (R521K), HRAS (G12S and R78W), and CASP8 (G328E).

\section{Integrated analysis identifies hallmark alterations in HNSCC cell lines}

The first step of integration analysis involved identification of genes with positively correlated copy number and expression data. While no significant correlation was observed among expression and arm-level copy number segments (Additional file 1: Figure S6a), median expression of focally amplified and deleted genes positively correlated to their expression (Fig. 2a and Additional file 1: Figure S6b). About 1000 genes with focal copy number changes with consistent expression pattern were identified from four cell lines. The second step of integration analysis involved identification of mutated genes that were expressed. Number of missense mutations identified from transcriptome sequencing $(67,641$ variants) were much higher than from exome sequencing (30,649 variants). Filtering of exome variants against transcriptome variants reduced total number of 9253 unique missesne variants in all four cell lines (Fig. 2b). Two thousand four hundred seventy-nine missense mutations of 9523 total mutations found across all cells were used for further integration with copy number and expression data 

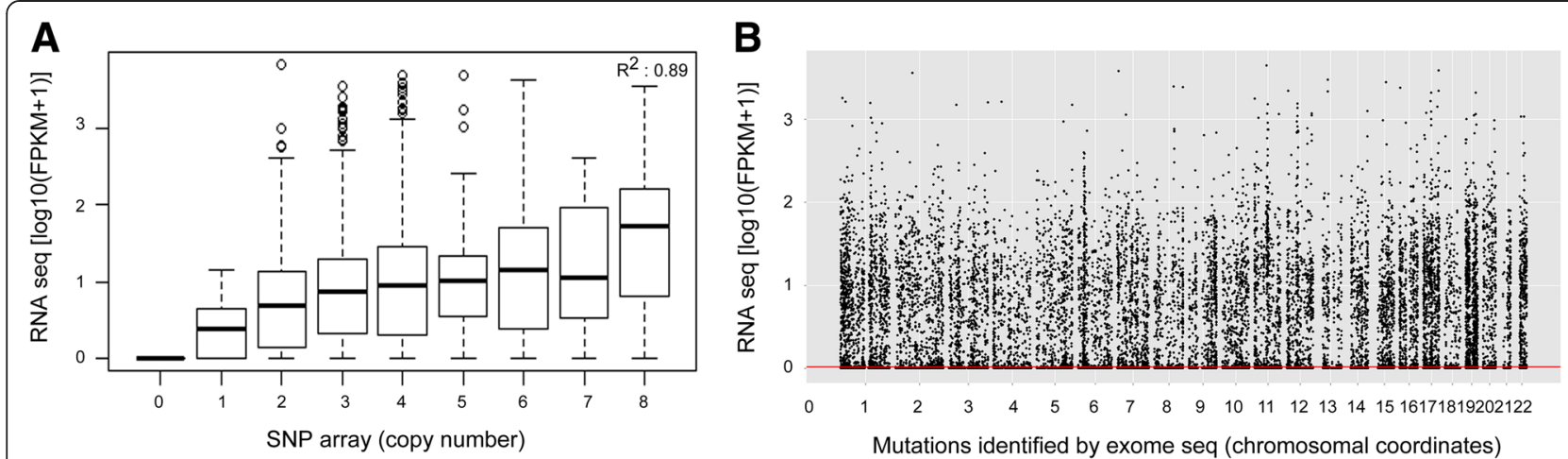

Fig. 2 Integration of copy-number, gene expression and single nucleotide variants. a Box plot representations showing focal copy number variations identified by SNP array on X-axis and gene expression quantified by RNA sequencing on Y-axis. Focally amplified or deleted genes from all four cell lines showed a positive trend with over expression or under expression respectively. $\mathbf{b}$ Scatter plot representation of genes harboring single nucleotide variants and it's expression from all four cell lines. Variants identified by whole exome sequencing are displayed by genomic coordinates on X-axis and normalized quantity of gene expression (by transcriptome sequencing) is displayed on Y-axis. Red line denotes 0.01 log 10 (FPKM +1 ) filter which removes $\sim 50 \%$ of the total variants

(Additional file 1: Figure S7). Next, as third step of integration, we sorted genes with altered copy number, expression levels and harboring non-synonymous mutations for integrated analysis based on criterion as described in methodology in four cell lines (Additional file 1: Figure S7). Briefly, genes harbouring two or more type of alterations were selected: harbouring positive correlation of focal copy number and gene expression; or those harbouring point mutations with detectable transcript harbouring the variant-based on which, we identified 38 genes having multiple types of alterations (Additional file 2: Table S6). These include genes known to have somatic incidences in HNSCC: TP53, HRAS, MET and PTEN. We also identified CASP8 in AW13516 cell line which was recently identified as very significantly altered by ICGC-India team in $\sim 50$ Indian HNSCC patients [50]. We additionally identified novel genes like CCNDBP1, GSN, IMMT, LAMA5, SAT2 and WDYHV1 to be altered by all three analysis i.e. CNV, expression and mutation. These all genes were also found to be altered in TCGA dataset with minimum $3 \%$ cumulative frequency (Additional file 1: Figure S8). The overall convergence of copy number, expression and mutation data in each cell line is represented as circos plot (Fig. 3a; Additional file 1: Figure S9). Among the novel genes identified, of genes with at least one identical mutation previously reported include a pseudokinase Nuclear receptor binding protein NRBP1 harboring heterozygous truncating mutation $\left(\mathrm{Q} 73^{*}\right)$ in NT8e cells, identical to as reported in lung cancer and altered in other cancers $[51,52]$.

\section{Mutant NRBP1 is required for tumor cell survival and is oncogenic in NIH3T3 cells}

NRBP1 encodes for three different nuclear receptor binding protein isoform using three alternative translational initiation sites of $60 \mathrm{kDa}, 51 \mathrm{kDa}$ and $43 \mathrm{kDa}$ [53], as were observed in 2 of 3 HNSCC cells (Fig. 4a). To determine whether expression of mutant NRBP1 is required for tumor cell survival, we tested shRNA constructs in two HNSCC cells expressing all three forms of WT NRBP1 (OT9 cells) and mutant NRBP1 (NT8e cells). We demonstrate that even partial knockdown of mutant NRBP1 expression in the NT8e cells, but not WT NRBP1 expression in the OT9, significantly inhibited anchorage-independent growth and cell survival (Fig. 4b-d). We next tested the oncogenic role of NRBP1. mRNAs harboring premature termination (nonsense) codons are selectively degraded by Nonsense-mediated mRNA decay (NMD) [54]. However, mRNAs with nonsense mutations in the first exon are known to bypass NMD [55]. When ectopically expressed in NIH-3 T3 cells, mutant NRBP1 transcript escape non-sense mediated degradation as determined by real time PCR (Additional file 1: Figure S10). All three isoform of NRBP1 were detected in NIH-3 T3 cells expressing wild type NRBP1 cDNA. However, only two isoform of $51 \mathrm{kDa}$ and $43 \mathrm{kDa}$ were detected in cells transfected with mutant NRBP1 cDNA (Fig. 4e upper panel). The over expression of the mutant NRBP1 in NIH3T3 cells conferred anchorage-independent growth, forming significantly higher colonies in soft agar than cells expressing wild type NRBP1 (Fig. 4f). Transformation of NIH-3 T3 cells by NRBP1 over expression was accompanied by elevated phosphorylation of the MAPK (Fig. 4e lower panel).

\section{Integrated analysis of TCGA dataset for HNSCC hallmark genes}

Next, as a proof of principle, we computated cumulative frequency of copy number variations, expression changes and point mutations across 43 genes with $\sim 3 \%$ 

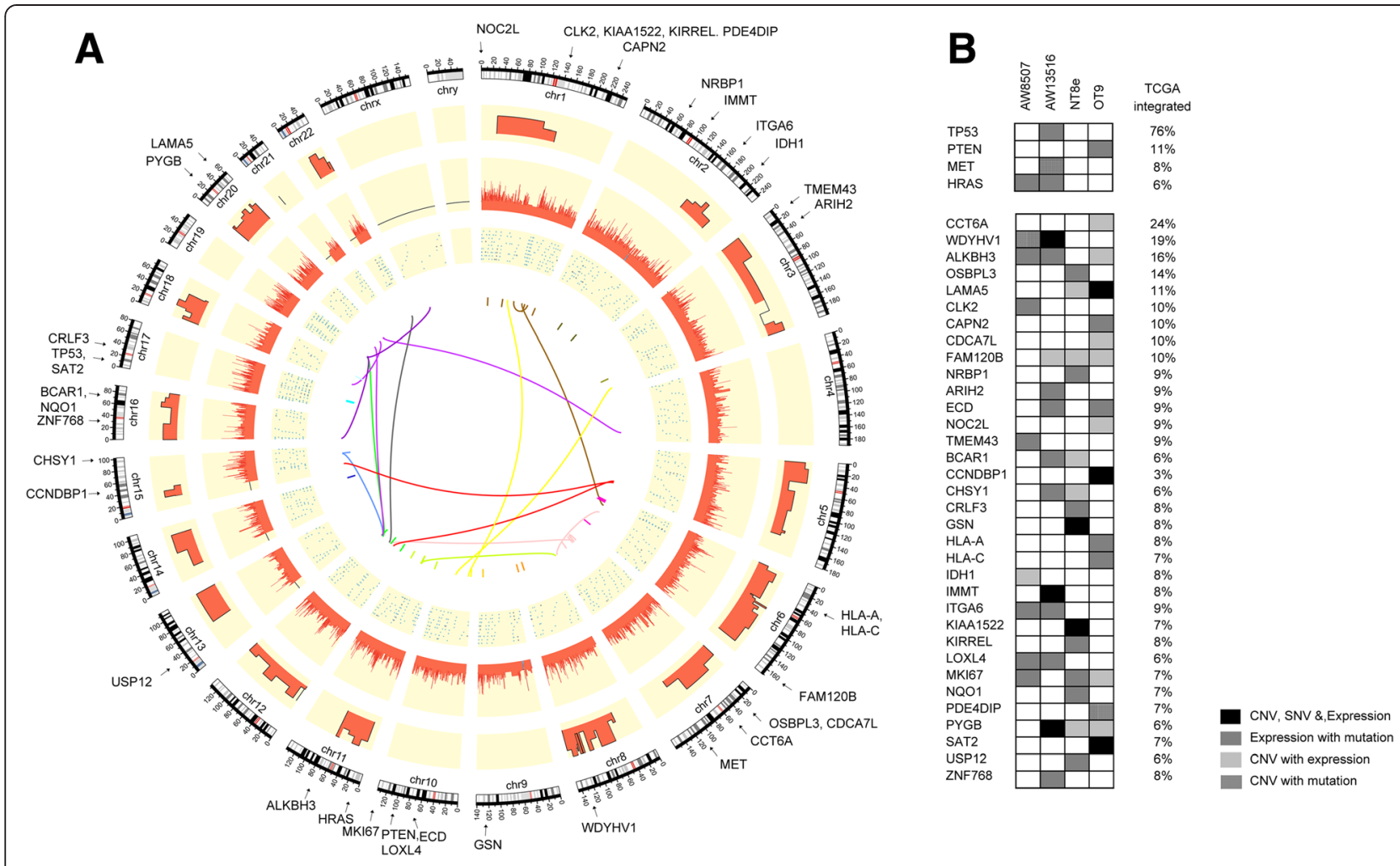

Fig. 3 Integrative genomic landscape of HNSCC. a Circos plot representations of genes identified by integrated genomic analysis of HNSCC cell lines. From outside to inside: karyotype, CNVs, Gene expression (FPKM), SNVs and translocations. Red colour indicates copy number gain or higher gene expression and blue colour indicates copy number loss or lower gene expression in CNV and FPKM tracks respectively. Non-synonymous mutations are indicated as blue triangles and grey circles represents non-sense mutations in SNV track. Fusion transcripts identified by transcriptome sequencing are shown as arc coloured by their chromosome of origin identified by ChimeraScan. $\mathbf{b}$ Heatmap representation of novel genes identified by integrated analysis of four HNSCC cell lines and their incidence in 279 HNSCC samples from TCGA study. Amplification (red) and deletions (blue) are indicated by filled box, over expression (red) and under expression (blue) are indicated by border line to the box, mis-sense (green), non-sense (black) and in-frame (brown) mutations are indicated by smaller square box

and higher mutation frequency in HNSCC TCGA dataset. As expected and described for few genes $[4,56]$, most of the genes were found to be altered at higher cumulative incidence than as reckoned by individual alterations (Fig. 5). Interestingly, three class of hallmark genes involved in HNSCC could be distinctly identified: genes that are primarily altered by mutations like TP53 and SYNE1; genes that are sparsely altered by amplification or overexpression in addition to mutations like FAT1, NOTCH1, KMT2D, and FLG; and, genes that are preferentially altered by amplification or over expression over point mutations with higher cumulative effect than known before. Of these, previously described genes like PIK3CA, CDKN2A, TP63, EGFR, CASP8, NFE2L2, and $K R A S$ show more than twice cumulative effect of alteration while rest of the genes are altered at several folds higher cumulative frequency based on integrated analysis. Furthermore, three genes- UBR5, ZNF384 and TERT were found to be altered with cumulative frequency of 32,19 , and $16 \%$, respectively that has not been previously described in HNSCC.

\section{Discussion}

We have characterized genetic alterations of unknown somatic status underlying four head and neck cancer cell lines of Indian origin patient by subjecting them to a thorough karyotype based characterization, SNP array based analysis, whole exome capture sequencing, and mRNA sequencing.

Integrated analysis of the cell lines establish their resemblance to primary tumors. Consistent with literature, most frequent copy number gains in head and neck cancer cells in this study were observed at $2 q, 3 q, 5 p$ and $7 \mathrm{p}$, and deletions at $3 \mathrm{p}, 9 \mathrm{p}, 10 \mathrm{p}, 11 \mathrm{q}, 14 \mathrm{q}, 17 \mathrm{q}$ and $19 \mathrm{p}$, as reported earlier $[57,58]$. Integration of multiple platform with the copy number variation, allowed us to identify the functionally relevant alterations including several hall marks genes known to be involved in HNSCC, viz. PIK3CA, EGFR, HRAS, MYC, CDKN2A, $M E T$, TRAF2, PTK2 and CASP8. Of the novel genes, $J A K 1$ was found to be amplified in two of the cell lines and overexpressed in all 4 HNSCC cells; NOTCH1 known to harbor inactivating mutations in $\operatorname{HNSCC}[3,50]$ was 


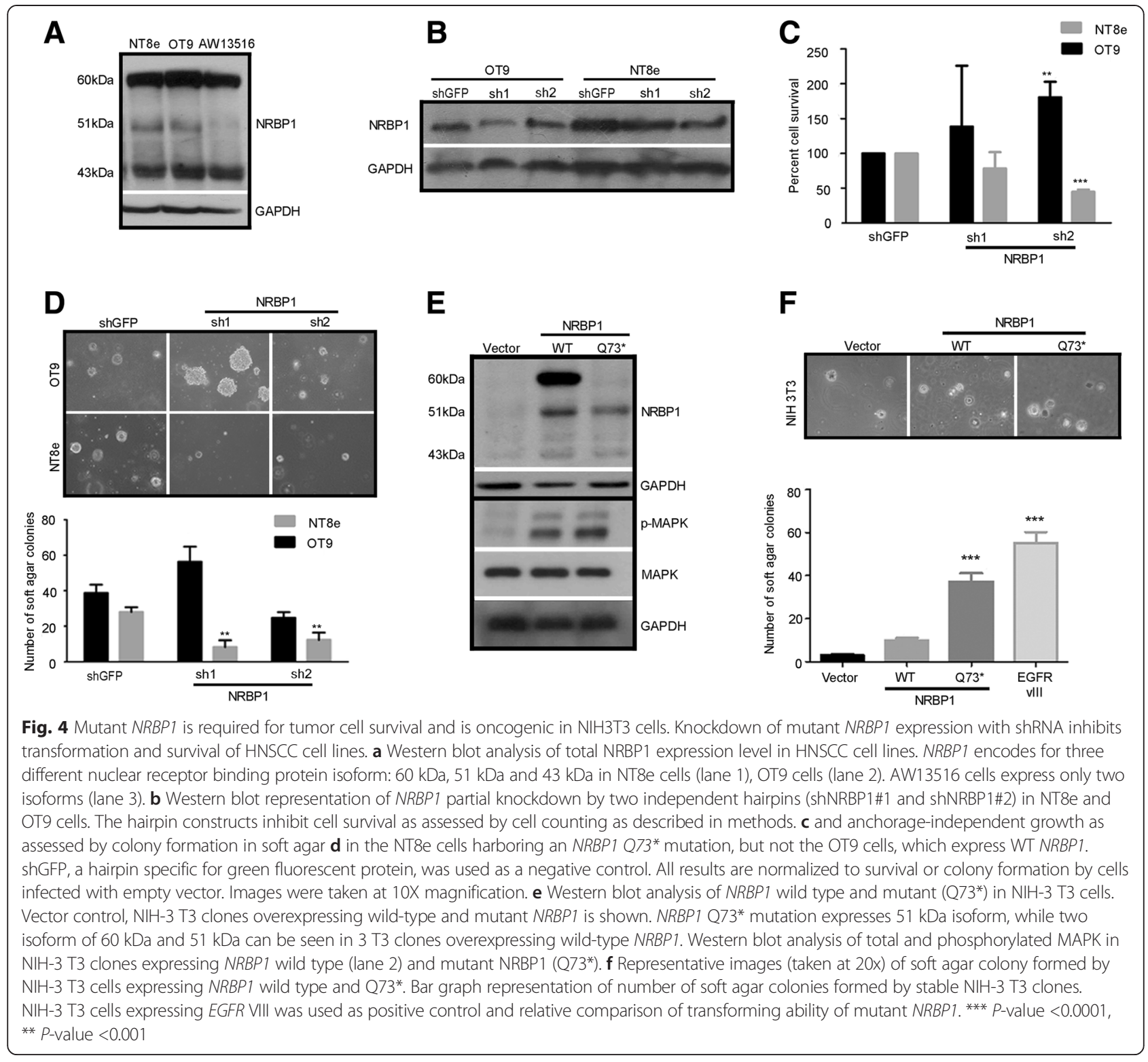

found to be amplified in all 4 and overexpressed in 2 of 4 HNSCC cells, known to be play dual role in a context dependent manner [59].

We also observed missense mutations in several novel genes such as CLK2, NRBP1, CCNDBP1, IDH1, LAMA5, $B C A R 1, Z N F 678$, and CLK2. Of these, genes with at least one identical mutation previously reported include NRBP1 (Q73*), a pseudo kinase, found in NT8e cells, earlier reported in lung and other cancers [51, 52], with an overall $9 \%$ cumulative frequency alteration in TCGA HNSCC dataset (Additional file 1: Figure S8). Of 48 pseudo kinases known in human genome, several have been shown to retain their biochemical catalytic activities despite lack of one or more of the three catalytic residues essential for its kinase activity, with their established roles in cancer [60-62].
Interestingly, several activating mutant alleles of NRBP1 homolog Drosophila Madm (Mlf1 adapter molecule) 3 T4 (Q46*); $2 U 3$ (C500*); $3 G 5\left(\mathrm{Q} 530^{*}\right) ; 7$ L2 and $3 Y 2$ (that disrupts splice donor site of first exon) are known, wherein alternative translation start codons is similarly suggestive for a varying degree of pinhead phenotype severity associated with the mutant alleles $[53,63]$. Studies in the fruit fly have provided important insights into mechanisms underlying the biology of growth promoting NRBP1 homolog Drosophila Madm. A recent study suggests Drosophila Madm interacts with Drosophila bunA that encodes a gene homologous to human Transforming Growth Factor- $\beta 1$ stimulated clone-22 TSC-22 [63]; that were later shown to interact even in mammalian system [64]. Interestingly, mammalian tumor suppressor TSC-22 is 


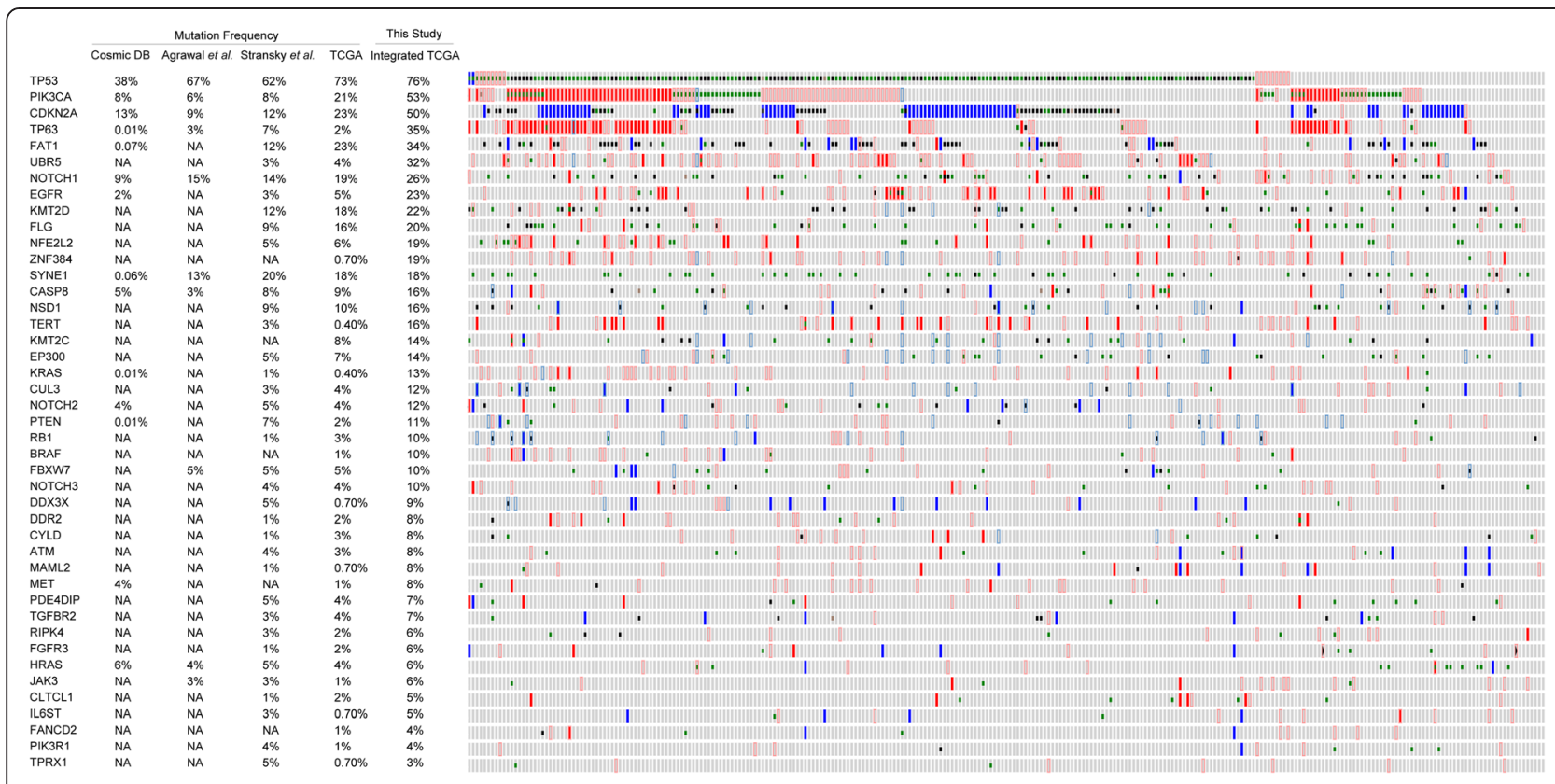

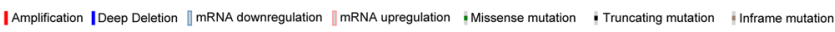

Fig. 5 Integrative genomic landscape of HNSCC tumors in TCGA dataset. Heatmap representation of hallmark genes in 279 HNSCC samples from TCGA study with frequency of alterations based on integrated CNVs, gene expression and SNVs. Frequency of alterations in the gene based on Cosmic database, Agrawal et al., Stransky et al. and TCGA study are also shown for comparison with integrated analysis. Amplification (red) and deletions (blue) are indicated by filled box, over expression (red) and under expression (blue) are indicated by border line to the box, mis-sense (green), non-sense (black) and in-frame (brown) mutations are indicated by smaller square box

known to play an important role in maintaining differentiated phenotype in salivary gland tumors [65], a subtype of head and neck cancer. More recently, studies have shown poor clinical outcomes are associated with NRBP1 over expression in prostate cancer [64]. We provide the first functional analysis of mutant NRBP1 and establish that NIH-3 T3 cells expressing the mutant NRBP1 enhance their survival and anchorage independent growth, while its knock down diminishes survival and anchorageindependent growth by oral cancer cells expressing activating NRBP1 mutations. Thus, NT8e cells harboring mutant NRBP1 was found to be consistent with its suggestive role in prostate cancer biology and other model organisms. Interestingly, $N R B P 1$ has also been shown to be involved in intestinal progenitor cell homeostasis with tumor suppressive function [66], suggesting its role is specific to the cellular context. This study identifies NRBP1 mutant to play an oncogenic role in head and neck cancer. However, in depth systematic sequencing of NRBP1 in a wide variety of tumor types may help indicate utility of NRBP1 inhibition in human cancer.

Furthermore, based on TCGA data integrated analysis, cumulative alteration frequency of TP63 (35\%), EGFR (23\%) and NFEL2 (19\%) were found to be higher than reported in COSMIC and cBioPortal, consistent with as described in other reports $[4,56]$. Of alterations not defined before, UBR5, ZNF384 and TERT were found to be altered at higher frequency at $32,19,16 \%$, respectively. Interestingly, recurrent UBR5-ZNF384 fusion has been shown to be oncogenic in EBV-associated nasopharyngeal subtype of HNSCC [67]; amplification of TERT has been shown to be higher in lung squamous [68], suggesting these alterations as potential squamous specific event, though that warrants detailed systematic assessment.

In overall, this study underscores integrative approaches through a filtering strategy to help reckon higher cumulative frequency for individual genes affected by two or more alterations to achieve the threshold for statistical significance even from fewer samples. The integrative analysis as described here, in essence, is based on a linear simplified assumption of disease aetiology that variation at DNA level lead to changes in gene expression causal to transformation of the cell. As a major deficiency, only genes that are subject to multiple levels of biological regulation are likely to be determined by this approach than genes that are primarily altered by single alteration like amplification or over expression.

\section{Conclusion}

As a proof of principle, integrated analysis of copy number variation, exome and transcriptome of 4 head 
and neck cancer cell lines and TCGA HNSCC dataset identify NRBP1, UBR5, ZNF384 and TERT as novel candidate oncogenes in HNSCC. However, systematic functional experimental validation is required to further guide and identify true driver events of these alterations. Additionally, the genetically- defined cellular systems characterized by integrated genomics analysis in this study (NT8e, OT9, AW13516, AW8507), together with the identification of novel actionable molecular targets, may help further facilitate the pre-clinical evaluation of emerging therapeutic agents in head and neck cancer.

\section{Additional files}

Additional file 1: Additional Figures S1-S10. Chromosomal aberration in HNSCC patient derived cell lines AW8507, AW13516, NT8e and OT9. (A) Representative karyotype of AW8507, AW13516, NT8e and OT9 cells is shown from total 25 karyotypes obtained per cell line. (B) Chromosomal aberrations identified by 25 independent karyotype of each cell line is represented in circular form. Chromosome numbers in each cell line are indicated by $\mathrm{n}$, as observed from karyotype $\left(^{*}\right)$ and predicted by SNP array $(\wedge)$. Copy number changes in HNSCC cell lines identified by SNP array. Genome alteration print (GAP) of (A) AW8507, (B) AW13516, (C) NT8e AND (D) OT9 cell lines obtained by SNP array. First horizontal block represents B-allele frequency, second block represents absolute copy number, third block is log $R$ ratio. Frequency of transcripts per binned log transformed FPKM + 1. Raw RNA sequencing data was binned to obtain frequency of genes per log10(FPKM + 1) in (A) AW8507, (B) AW13516, (C) NT8e and (D) OT9. Horizontal dotted lines indicates percentile of transcripts in the quadrant. Similarity of gene expression in HNSCC cell lines. Number of genes commonly expressed between AW8507, AW13516, NT8e and OT9 cell lines. Relative depth in exome sequencing. Relative depth of sequencing for various genomic regions in (A) AW8507, (B) AW13516, (C) NT8e and (D) OT9. Correlation of copy number with gene expression. (A) Arm level and (B) focal copy number changes and gene expression (y-axis) are shown for AW8507, AW13516, NT8e and OT9 cell lines. Correlation of focal copy number with gene expression was 1.5 fold higher in AW8507, 5.2 fold higher in AW13516, 2.4 fold higher in NT8e and 1.6 fold higher in OT9 cell line compare to arm level copy number changes. $P$-value cut-off of 0.05 was used as threshold for statistical significance. ${ }^{*}$ denotes $P$-value $<0.05,{ }^{* *}<0.005,{ }^{* * *}<0.0005$. Schematic view of data reduction in integrated genomic analysis. Flow chart depicts the reduction of data at each stage of integration. First row indicates number of genes identified from each platform as raw calls. Second and third row indicates number of genes left after each step of integration with main selection parameter indicated outside the box. Integrative genomic alterations of genes in TCGA dataset of HNSCC tumors. Heatmap representation of 38 genes in 279 HNSCC samples from TCGA study with frequency of alterations based on integrated CNVs, gene expression and SNVs. Amplification (red) and deletions (blue) are indicated by filled box, over expression (red) and under expression (blue) are indicated by border line to the box, mis-sense (green), non-sense (black) and in-frame (brown) mutations are indicated by smaller square box. Circos plot representation of HNSCC cell lines. Circos plot representations of integrated genomic data of (A) AW8507, (B) AW13516, (C) NT8e and (D) OT9 cell lines. From outside to inside: karyotype, CNVs, Gene expression (FPKM), SNVs and translocations. Red colour indicates copy number gain or higher gene expression and blue colour indicates copy number loss or lower gene expression in CNV and FPKM tracks, respectively. Non-synonymous mutations are indicated as blue triangles and grey circles represents non-sense mutations in SNV track. Fusion transcripts identified by transcriptome sequencing are shown as arc coloured by their chromosome of origin identified by ChimeraScan. NRBP1 expression in NIH-3 T3 cells. QPCR analysis of NRBP1 gene expression in $\mathrm{NIH}-3 \mathrm{~T} 3$ stably expressing wild and mutant. Data was normalized against GAPDH and fold change plotted. $P$ value $<0.0001$ is denoted as ${ }^{* * *}$. (PDF $9017 \mathrm{~kb}$ )
Additional file 2: Additional Tables S1-S8: Copy number alterations of known genomic locations identified in HNSCC cell lines. Copy number alterations in halmark genes identified in HNSCC cell lines. Gene expression of hallmark genes by RNA sequencing and qPCR. Features of whole exome and transcriptome sequencing. Validation of mutations in hallmark and novel genes. Details of mutations identified by integrated analysis in HNSCC cell lines. Primer sequences used for Sanger sequenicng based validation of mutations. Primers used for copy number and gene expression study using qPCR. (ZIP $249 \mathrm{~kb})$

\section{Abbreviations}

HNSCC: Head and neck squamous cell carcinoma; COSMIC: Catalogue of Somatic Mutation In Cancer; TCGA: The Cancer Genome Atlas;

ICGC: International Cancer Genome Consortium; CIN: Chrmosomal Instability; CNV: Copy Number Variation.

\section{Competing interests}

The authors declare that they have no competing interests.

\section{Authors' contributions}

PC and PU contributed equally to this work; PC, PU, SG, and $\mathrm{AD}$ designed research; PC, PU, PI, MT, MS, GVR, NO, AK, RC, and MR performed research; PC, PU, MS, and NO contributed new reagents/analytic tools; PC, PU, PI, MT, MS, GVR, NO, AK, RC, MR and AD analyzed data; and PC, PU, SG and AD wrote the paper. All authors have read and approved the manuscript.

\section{Acknowledgements}

All members of the Dutt laboratory for critically reviewing the manuscript. Rita Mulherkar for establishing and sharing OT9 and NT8e cells. Anti Cancer Drug Screening Facility at ACTREC, Tata Memorial Center for AW13516 and AW8507 cells. Genotypic Inc., Sandor Proteomics Pvt. Ltd. and Centre for Cellular and Molecular Platforms (C-CAMP), for providing sequencing and SNP array genotyping services. A.D. is supported by an Intermediate Fellowship from the Wellcome Trust/DBT India Alliance (IA///11/2500278), by a grant from DBT (BT/PR2372/AGR/36/696/2011), and intramural grants (IRB project 92 and 55). P.C. and P.I are supported by senior research fellowship from ACTREC. P.U. is supported by senior research fellowship from CSIR. The funders had no role in study design, data collection and analysis, decision to publish, or preparation of the manuscript.

\section{Author details}

'Advanced Centre for Treatment, Research and Education in Cancer, Tata Memorial Center, Navi Mumbai, Maharashtra 410210, India. ${ }^{2}$ Department of Medical Oncology, Tata Memorial Hospital, Tata Memorial Center, Mumbai, Maharashtra, India.

Received: 13 August 2015 Accepted: 23 October 2015

Published online: 14 November 2015

\section{References}

1. Rothenberg SM, Ellisen LW. The molecular pathogenesis of head and neck squamous cell carcinoma. J Clin Invest. 2012;122(6):1951-7.

2. Agrawal N, Frederick MJ, Pickering CR, Bettegowda C, Chang K, Li RJ, et al. Exome sequencing of head and neck squamous cell carcinoma reveals inactivating mutations in NOTCH1. Science. 2011;333(6046):1154-7.

3. Stransky N, Egloff AM, Tward AD, Kostic AD, Cibulskis K, Sivachenko A, et al. The mutational landscape of head and neck squamous cell carcinoma. Science. 2011;333(6046):1157-60.

4. Cancer Genome Atlas N. Comprehensive genomic characterization of head and neck squamous cell carcinomas. Nature. 2015;517(7536):576-82.

5. Lawrence MS, Stojanov P, Polak P, Kryukov GV, Cibulskis K, Sivachenko A, et al. Mutational heterogeneity in cancer and the search for new cancerassociated genes. Nature. 2013;499(7457):214-8.

6. Dees ND, Zhang Q, Kandoth C, Wendl MC, Schierding W, Koboldt DC, et al. MuSiC: identifying mutational significance in cancer genomes. Genome Res. 2012;22(8):1589-98.

7. Gonzalez-Perez A, Perez-Llamas C, Deu-Pons J, Tamborero D, Schroeder MP, Jene-Sanz A, et al. IntOGen-mutations identifies cancer drivers across tumor types. Nat Methods. 2013;10(11):1081-2.

8. Hodis E, Watson IR, Kryukov GV, Arold ST, Imielinski M, Theurillat JP, et al. A landscape of driver mutations in melanoma. Cell. 2012;150(2):251-63. 
9. Reimand J, Bader GD. Systematic analysis of somatic mutations in phosphorylation signaling predicts novel cancer drivers. Mol Syst Biol. 2013;9:637.

10. Mermel CH, Schumacher SE, Hill B, Meyerson ML, Beroukhim R, Getz G. GISTIC2.0 facilitates sensitive and confident localization of the targets of focal somatic copy-number alteration in human cancers. Genome Biol. 2011;12(4):R41.

11. Lawrence MS, Stojanov P, Mermel CH, Robinson JT, Garraway LA, Golub TR, et al. Discovery and saturation analysis of cancer genes across 21 tumour types. Nature. 2014;505(7484):495-501.

12. Akavia UD, Litvin O, Kim J, Sanchez-Garcia F, Kotliar D, Causton HC, et al. An integrated approach to uncover drivers of cancer. Cell. 2010;143(6):1005-17.

13. Upadhyay $P$, Dwivedi R, Dutt A. Applications of next-generation sequencing in cancer. Curr Sci. 2014;107(5):795.

14. Natrajan R, Wilkerson P. From integrative genomics to therapeutic targets. Cancer Res. 2013;73(12):3483-8.

15. Pickering CR, Zhang J, Yoo SY, Bengtsson L, Moorthy S, Neskey DM, et al. Integrative genomic characterization of oral squamous cell carcinoma identifies frequent somatic drivers. Cancer Discov. 2013;3(7):770-81.

16. Wilkerson MD, Cabanski CR, Sun W, Hoadley KA, Walter $V$, Mose LE, et al. Integrated RNA and DNA sequencing improves mutation detection in low purity tumors. Nucleic Acids Res. 2014;42(13):e107.

17. Kristensen VN, Lingjaerde OC, Russnes HG, Vollan HK, Frigessi A, BorresenDale AL. Principles and methods of integrative genomic analyses in cancer. Nat Rev Cancer. 2014;14(5):299-313.

18. Mulherkar R, Goud AP, Wagle AS, Naresh KN, Mahimkar MB, Thomas SM, et al. Establishment of a human squamous cell carcinoma cell line of the upper aero-digestive tract. Cancer Lett. 1997;118(1):115-21.

19. Tatake RJ, Rajaram N, Damle RN, Balsara B, Bhisey AN, Gangal SG. Establishment and characterization of four new squamous cell carcinoma cell lines derived from oral tumors. J Cancer Res Clin Oncol. 1990;116(2):179-86.

20. Popova T, Manie E, Stoppa-Lyonnet D, Rigaill G, Barillot E, Stern MH. Genome Alteration Print (GAP): a tool to visualize and mine complex cancer genomic profiles obtained by SNP arrays. Genome Biol. 2009;10(11):R128.

21. Quinlan AR, Hall IM. BEDTools: a flexible suite of utilities for comparing genomic features. Bioinformatics. 2010;26(6):841-2.

22. Li H, Durbin R. Fast and accurate short read alignment with BurrowsWheeler transform. Bioinformatics. 2009:25(14):1754-60.

23. McKenna A, Hanna M, Banks E, Sivachenko A, Cibulskis K, Kernytsky A, et al. The Genome Analysis Toolkit: a MapReduce framework for analyzing next-generation DNA sequencing data. Genome Res. 2010;20(9):1297-303.

24. DePristo MA, Banks E, Poplin R, Garimella KV, Maguire JR, Hartl C, et al. A framework for variation discovery and genotyping using next-generation DNA sequencing data. Nat Genet. 2011:43(5):491-8.

25. Cibulskis K, Lawrence MS, Carter SL, Sivachenko A, Jaffe D, Sougnez C, et al. Sensitive detection of somatic point mutations in impure and heterogeneous cancer samples. Nat Biotechnol. 2013;31(3):213-9.

26. Sherry ST, Ward MH, Kholodov M, Baker J, Phan L, Smigielski EM, et al. dbSNP: the NCBI database of genetic variation. Nucleic Acids Res. 2001;29(1):308-11.

27. Forbes SA, Bhamra G, Bamford S, Dawson E, Kok C, Clements J, et al. The Catalogue of Somatic Mutations in Cancer (COSMIC). Curr Protoc Hum Genet. 2008;Chapter 10:Unit 1011.

28. Ramos AH, Lichtenstein L, Gupta M, Lawrence MS, Pugh TJ, Saksena G, et al. Oncotator: cancer variant annotation tool. Hum Mutat. 2015;36(4):E2423-9.

29. Adzhubei I, Jordan DM, Sunyaev SR. Predicting functional effect of human missense mutations using PolyPhen-2. Curr Protoc Hum Genet. 2013; Chapter 7:Unit7 20.

30. Choi $Y$, Chan AP. PROVEAN web server: a tool to predict the functional effect of amino acid substitutions and indels. Bioinformatics. 2015:31(16):2745-2747.

31. Reva B, Antipin $Y$, Sander C. Predicting the functional impact of protein mutations: application to cancer genomics. Nucleic Acids Res. 2011;39(17):e118.

32. Kim D, Pertea G, Trapnell C, Pimentel H, Kelley R, Salzberg SL. TopHat2: accurate alignment of transcriptomes in the presence of insertions, deletions and gene fusions. Genome Biol. 2013;14(4):R36.

33. Trapnell C, Roberts A, Goff L, Pertea G, Kim D, Kelley DR, et al. Differential gene and transcript expression analysis of RNA-seq experiments with TopHat and Cufflinks. Nat Protoc. 2012;7(3):562-78.
34. Barbieri CE, Baca SC, Lawrence MS, Demichelis F, Blattner M, Theurillat JP, et al. Exome sequencing identifies recurrent SPOP, FOXA1 and MED12 mutations in prostate cancer. Nat Genet. 2012;44(6):685-9.

35. Subramanian A, Tamayo P, Mootha VK, Mukherjee S, Ebert BL, Gillette MA, et al. Gene set enrichment analysis: a knowledge-based approach for interpreting genome-wide expression profiles. Proc Natl Acad Sci U S A. 2005;102(43):15545-50.

36. Iyer MK, Chinnaiyan AM, Maher CA. ChimeraScan: a tool for identifying chimeric transcription in sequencing data. Bioinformatics. 2011;27(20):2903-4.

37. Krzywinski M, Schein J, Birol I, Connors J, Gascoyne R, Horsman D, et al. Circos: an information aesthetic for comparative genomics. Genome Res. 2009;19(9):1639-45.

38. Minton JA, Flanagan SE, Ellard S. Mutation surveyor: software for DNA sequence analysis. Methods Mol Biol. 2011;688:143-53.

39. Livak KJ, Schmittgen TD. Analysis of relative gene expression data using real-time quantitative PCR and the 2(-Delta Delta C(T)) Method. Methods. 2001;25(4):402-8.

40. Sancak Y, Peterson TR, Shaul YD, Lindquist RA, Thoreen CC, Bar-Peled L, et al. The Rag GTPases bind raptor and mediate amino acid signaling to mTORC1. Science. 2008;320(5882):1496-501.

41. Dutt A, Salvesen HB, Chen TH, Ramos AH, Onofrio RC, Hatton C, et al. Drug-sensitive FGFR2 mutations in endometrial carcinoma. Proc Natl Acad Sci U S A. 2008;105(25):8713-7.

42. Moffat J, Grueneberg DA, Yang X, Kim SY, Kloepfer AM, Hinkle G, et al. A lentiviral RNAi library for human and mouse genes applied to an arrayed viral high-content screen. Cell. 2006;124(6):1283-98.

43. Walker JM. The bicinchoninic acid (BCA) assay for protein quantitation Methods Mol Biol. 1994;32:5-8.

44. Roschke AV, Tonon G, Gehlhaus KS, McTyre N, Bussey KJ, Lababidi S, et al. Karyotypic complexity of the NCl-60 drug-screening panel. Cancer Res. 2003;63(24):8634-47.

45. Yamamoto N, Mizoe J, Numasawa H, Tsujii H, Shibahara T, Noma H. Allelic loss on chromosomes 2q, 3p and 21q: possibly a poor prognostic factor in oral squamous cell carcinoma. Oral Oncol. 2003:39(8):796-805.

46. Partridge M, Emilion G, Langdon JD. LOH at $3 p$ correlates with a poor survival in oral squamous cell carcinoma. Br J Cancer. 1996;73(3):366-71.

47. Meredith SD, Levine PA, Burns JA, Gaffey MJ, Boyd JC, Weiss LM, et al. Chromosome 11q13 amplification in head and neck squamous cell carcinoma. Association with poor prognosis. Arch Otolaryngol Head Neck Surg. 1995;121(7):790-4.

48. Chen Y, Chen C. DNA copy number variation and loss of heterozygosity in relation to recurrence of and survival from head and neck squamous cell carcinoma: a review. Head Neck. 2008;30(10):1361-83.

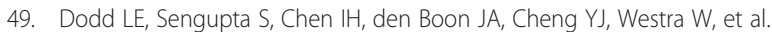
Genes involved in DNA repair and nitrosamine metabolism and those located on chromosome 14q32 are dysregulated in nasopharyngeal carcinoma. Cancer Epidemiol Biomarkers Prev. 2006:15(11):2216-25.

50. India Project Team of the International Cancer Genome C. Mutational landscape of gingivo-buccal oral squamous cell carcinoma reveals new recurrently-mutated genes and molecular subgroups. Nat Commun. 2013:4:2873.

51. Barretina J, Caponigro G, Stransky N, Venkatesan K, Margolin AA, Kim S, et al. The Cancer Cell Line Encyclopedia enables predictive modelling of anticancer drug sensitivity. Nature. 2012;483(7391):603-7.

52. Davies H, Hunter C, Smith R, Stephens P, Greenman C, Bignell G, et al. Somatic mutations of the protein kinase gene family in human lung cancer. Cancer Res. 2005;65(17):7591-5.

53. Hooper JD, Baker E, Ogbourne SM, Sutherland GR, Antalis TM. Cloning of the CDNA and localization of the gene encoding human NRBP, a ubiquitously expressed, multidomain putative adapter protein. Genomics. 2000;66(1):113-8

54. Schweingruber C, Rufener SC, Zund D, Yamashita A, Muhlemann O. Nonsense-mediated mRNA decay - mechanisms of substrate mRNA recognition and degradation in mammalian cells. Biochim Biophys Acta. 2013;1829(6-7):612-23.

55. Neu-Yilik G, Amthor B, Gehring NH, Bahri S, Paidassi H, Hentze MW, et al. Mechanism of escape from nonsense-mediated mRNA decay of human beta-globin transcripts with nonsense mutations in the first exon. RNA 2011;17(5):843-54

56. Rusan M, Li YY, Hammerman PS. Genomic landscape of human papillomavirus-associated cancers. Clin Cancer Res. 2015;21(9):2009-19. 
57. Smeets SJ, Braakhuis BJ, Abbas S, Snijders PJ, Ylstra B, van de Wiel MA, et al. Genome-wide DNA copy number alterations in head and neck squamous cell carcinomas with or without oncogene-expressing human papillomavirus. Oncogene. 2006;25(17):2558-64.

58. Ambatipudi S, Gerstung M, Gowda R, Pai P, Borges AM, Schäffer AA, et al. Genomic profiling of advanced-stage oral cancers reveals chromosome 11q alterations as markers of poor clinical outcome. PLoS ONE. 2011;6(2):e17250.

59. Ntziachristos P, Lim JS, Sage J, Aifantis I. From fly wings to targeted cancer therapies: a centennial for notch signaling. Cancer Cell. 2014;25(3):318-34.

60. Hua F, Mu R, Liu J, Xue J, Wang Z, Lin H, et al. TRB3 interacts with SMAD3 promoting tumor cell migration and invasion. J Cell Sci. 2011;124(Pt 19):3235-46.

61. Manning G, Whyte DB, Martinez R, Hunter T, Sudarsanam S. The protein kinase complement of the human genome. Science. 2002;298(5600):1912-34

62. Zeqiraj E, Filippi BM, Deak M, Alessi DR, van Aalten DM. Structure of the LKB1-STRAD-MO25 complex reveals an allosteric mechanism of kinase activation. Science. 2009;326(5960):1707-11.

63. Gluderer S, Oldham S, Rintelen F, Sulzer A, Schutt C, Wu X, et al. Bunched, the Drosophila homolog of the mammalian tumor suppressor TSC-22, promotes cellular growth. BMC Dev Biol. 2008:8:10.

64. Ruiz C, Oeggerli M, Germann M, Gluderer S, Stocker H, Andreozzi M, et al. High NRBP1 expression in prostate cancer is linked with poor clinical outcomes and increased cancer cell growth. Prostate. 2012;72(15):1678-87.

65. Doi Y, Kawamata H, Ono Y, Fujimori T, Imai Y. Expression and cellular localization of TSC-22 in normal salivary glands and salivary gland tumors: implications for tumor cell differentiation. Oncol Rep. 2008;19(3):609-16.

66. Wilson $\mathrm{CH}$, Crombie $\mathrm{C}$, van der Weyden L, Poulogiannis $\mathrm{G}$, Rust AG, Pardo M, et al. Nuclear receptor binding protein 1 regulates intestinal progenitor cell homeostasis and tumour formation. EMBO J. 2012:31(11):2486-97.

67. Chung GT, Lung RW, Hui AB, Yip KY, Woo JK, Chow C, et al. Identification of a recurrent transforming UBR5-ZNF423 fusion gene in EBV-associated nasopharyngeal carcinoma. J Pathol. 2013;231(2):158-67.

68. Zhu CQ, Cutz JC, Liu N, Lau D, Shepherd FA, Squire JA, et al. Amplification of telomerase (hTERT) gene is a poor prognostic marker in non-small-cell lung cancer. Br J Cancer. 2006;94(10):1452-9.

\section{Submit your next manuscript to BioMed Central and take full advantage of:}

- Convenient online submission

- Thorough peer review

- No space constraints or color figure charges

- Immediate publication on acceptance

- Inclusion in PubMed, CAS, Scopus and Google Scholar

- Research which is freely available for redistribution 